\title{
Protective effects of quercetin from oxidative/ nitrosative stress under intermittent hypobaric hypoxia exposure in the rat's heart
}

\author{
IC Chiş ${ }^{1}$, D Baltaru ${ }^{2}$, A Dumitrovici ${ }^{3}$, A Coseriu ${ }^{1}$, BC Radu ${ }^{1}$, R Moldovan ${ }^{1}$, \\ A Mureşan ${ }^{1}$
}

${ }^{1}$ Department of Physiology, "Iuliu Hatieganu" University of Medicine and Pharmacy, Cluj-Napoca, Romania

${ }^{2}$ Department of Internal Medicine, “Constantin Papilian” Military Emergency Hospital, Cluj-Napoca, Romania

${ }^{3}$ Department of Medical Oncology, "I. Chiricuta” Oncologic Institute, Cluj-Napoca, Romania

\author{
Received: October 23, 2017
}

Accepted: June 21, 2018

\begin{abstract}
Background: Exposure to high altitude in hypobaric hypoxia $(\mathrm{HH})$ is considered to be a physiological oxidative/ nitrosative stress. Quercetin (Que) is an effective antioxidant and free radical scavenger against oxidative/nitrosative stress. Aims: The aim of this study was to investigate the cardioprotective effects of Que in animals exposed to intermittent $\mathrm{HH}(\mathrm{IHH})$ and therefore exposed to oxidative/nitrosative stress. Materials and methods: Wistar albino male rats were exposed to short-term (2 days) or long-term (4 weeks; 5 days/week) IHH in a hypobaric chamber $\left(5,500 \mathrm{~m}, 8 \mathrm{~h} /\right.$ day, $380 \mathrm{mmHg}, 12 \% \mathrm{O}_{2}$, and $\left.88 \% \mathrm{~N}_{2}\right)$. Half of the animals received natural antioxidant Que (body weight: $30 \mathrm{mg} / \mathrm{kg}$ ) daily before each IHH exposure and the remaining rats received vehicle (carboxymethylcellulose solution). Control rats were kept under normobaric normoxia $(\mathrm{Nx})$ and treated in a corresponding manner. One day after the last exposure to IHH, we measured the cardiac hypoxia-induced oxidative/nitrosative stress biomarkers: the malondialdehyde (MDA) level and protein carbonyl (PC) content, the activity of some antioxidant enzymes [superoxide dismutase (SOD) and catalase (CAT)], the nitrite plus nitrate (NOx) production, and the inducible nitric oxide synthase (iNOS) protein expression. Results: Heart tissue MDA and PC levels, NOx level, and iNOS expression of IHH-exposed rats had increased, and SOD and CAT activities had decreased compared with those of the Nx-exposed rats (control groups). MDA, CP, NOx, and iNOS levels had decreased in Que-treated IHH-exposed rats compared with IHH-exposed rats (control groups). However, Que administration increased SOD and CAT activities of the heart tissue in the IHH-exposed rats. Conclusion: $\mathrm{HH}$ exposure increases oxidative/nitrosative stress in heart tissue and Que is an effective cardioprotective agent, which further supports the oxidative cardiac dysfunction induced by hypoxia.
\end{abstract}

Keywords: cardioprotection, intermittent hypobaric hypoxia, quercetin, oxidative/nitrosative stress, natural antioxidants

\section{Introduction}

Oxidative/nitrosative stress is involved both in pathological processes and in physiological processes, such as anaerobic physical exercise, pregnancy, aging, exposure to high altitude (HA), etc. (13). HA exposure decreases the partial pressure of oxygen and increases production of reactive oxygen and nitrogen species (RONS) by altering mitochondria electron transport chains, activating enzymes, such as nicotinamide adenine dinucleotide

Corresponding author: Irina Camelia Chis, MD, $\mathrm{PhD}$

Department of Physiology, "Iuliu Hatieganu" University of Medicine and Pharmacy

RO 400023, Clinicilor Street 1-3, Cluj-Napoca, Romania

Phone: +40 744707 080; Fax: +40 264597 257; E-mail: irinnaus@yahoo.com 
phosphate (NADPH) oxidase, xanthine oxidase/reductase, nitric oxide synthases (NOSs), etc., as well as by the depletion of cellular enzymatic and non-enzymatic antioxidants $(5,12$, 30). In high concentrations, RONS determines the expression of hypoxia-inducible factor-1 alpha (HIF-1 $\alpha$ ), which then activates over 100 genes involved inclusively in erythropoiesis, angiogenesis, energy metabolism, proliferation/cell survival, apoptosis, etc., and increases the expression of inducible NOS (iNOS) $(5,23,30)$.

In conditions of hypobaric hypoxia $(\mathrm{HH})$, the nuclear factor kappa $\mathrm{B}(\mathrm{NF}-\kappa \mathrm{B})$ is activated, leading to an increase in the expression of HIF-1 $\alpha$, which in turn increases the synthesis of iNOS and generation of nitric oxide (NO) $(30,41)$.

The heart is a vital organ for the body that has a strictly aerobic metabolism and is therefore very vulnerable to low levels of oxygen. Hypoxia induces cardiac apoptosis by the expression and accumulation of proapoptotic proteins, followed by cardiac remodeling, biventricular fibrosis, ventricular hypertrophy, left ventricular dysfunction, and heart failure. Excessive RONS production in the myocardium under the $\mathrm{HH}$ exposure conditions induces cardiomyocytes injury, affecting the structural and functional integrity of the heart $(25,26)$.

Exposure to long-term or short-term intermittent $\mathrm{HH}$ ( $\mathrm{IHH}$ ) has been reported to induce cardiac damage or cardiac protection in different studies under various conditions (36-40). Exposure to IHH has the same mechanism of myocardial ischemia/reperfusion injury and is associated with an increase in RONS production, which is generated during the phase of reoxygenation of IHH (16). Alteration of NO production plays an important role in heart injury in conditions of hypoxia/reoxygenation. Excessive NO production by the action of iNOS in the myocardium rapidly reacts with superoxide anion to form peroxynitrite (ONOO-), a reactive short-lived peroxide that affects mitochondrial function and triggers cell death via oxidation and nitration reactions. Peroxynitrite causes myocardial injury and endothelial dysfunction $(1,22,31)$. Controversial effects of $\mathrm{IHH}$ on cardiac damage or cardiac protection are still mysterious.

Flavonoids represent a large group of polyphenolic natural antioxidants present in large numbers in fruit, vegetables, spices, seeds, red wine, chocolate, etc. $(18,19,34)$. Quercetin (Que) is an essential flavonoid known to be a multifunctional agent due to its antioxidant and anti-inflammatory activity $(4,7)$.

Recent research has shown in in vitro and in vivo models that Que is an effective antioxidant by direct scavenge of RONS, including superoxide anion, NO, and peroxynitrite, and by increasing the endogenous antioxidant activity $(4,19,21,27)$. Several recent researches have shown that Que has protective effects on different types of cells, including cardiomyocytes, neurons, testis, renal, and liver cells in ischemia/reperfusion injury $(2-4,6,32)$.

This study investigates the potential cardioprotective role of Que in $\mathrm{HH}$-induced cardiomyocyte injury. We have focused on the correlation between Que administration in cardiomyocytes and the cardioprotective role of Que conducted by decreasing oxidative/ nitrosative stress induced in the heart by $\mathrm{HH}$ exposure.

\section{Materials and Methods}

\section{Drugs and chemicals}

Que was extracted, dosed, and encapsulated at "PROPLANTA" Applied Vegetal Biotechnologies Center in Cluj-Napoca, Romania. Que was standardized by its chemical composition using high-performance liquid chromatography method to ensure batch-to-batch consistency. The Que was suspended in $0.5 \%$ carboxymethylcellulose (CMC) solution as a vehicle. 


\section{Animals}

Sixty healthy Wistar albino male rats (3-month-old) were used in this study. The rats were purchased from the Experimental Animal House of the Faculty of Medicine within "Iuliu Hatieganu" University of Medicine and Pharmacy of Cluj-Napoca, Romania. The rats' mean weight was 260-325 g at the beginning of the trials. All rats used in the experiment were kept for 10 days to acclimatize to the conditions of the Animal House Laboratory at the Physiology Department before being introduced in the study. Throughout the entire period of the experiment, all rats were maintained in special cages artificially illuminated (12-h light-dark cycle), at a temperature of $21-23{ }^{\circ} \mathrm{C}$ and at $50 \%-60 \%$ humidity in the animal room.

The rats were given standard rat pellet diet and water ad libitum. All the experiments were performed according to the approved animal protocols of the Ethical Committee on Animal Welfare of "Iuliu Hatieganu" University in accordance with the Romanian Ministry of Health and complying with Guidelines in the Use of Animals in Toxicology.

\section{IHH-adapted rat model}

The IHH treatment consisted of intermittent exposure of Wistar rats to $\mathrm{HH}$ (equivalent to an altitude of 5,500 $\mathrm{m}$, barometric pressure $380 \mathrm{mmHg}, 12 \% \mathrm{O}_{2}$, and $88 \% \mathrm{~N}_{2}$ ) in a hypobaric chamber (of the Animal House Laboratory at the Physiology Department) for 2 days [shortterm IHH (STIHH) for $8 \mathrm{~h} /$ day] and for 4 consecutive weeks [long-term IHH (LTIHH), for $8 \mathrm{~h} /$ day, 5 days/week]. The rats were taken out of the hypobaric chamber once after every $8 \mathrm{~h}$ exposure in order to receive water and a standard laboratory diet.

The control rats maintained in normobaric normoxia $(\mathrm{Nx})$ conditions (barometric pressure $760 \mathrm{mmHg}, 21 \% \mathrm{O}_{2}$, and $79 \% \mathrm{~N}_{2}$ ) for a corresponding period, and were kept in the same environment as IHH rats with free access to water and food except for hypoxic exposure.

\section{Experimental design}

The rats were randomly divided into following six experimental groups $(n=10)$. First group (control group, STNx + CMC) - rats maintained in Nx conditions for short-term and treated with CMC; second group (control group, STIHH + CMC) - rats maintained in STIHH conditions and treated with CMC; third group (STIHH + Que) - rats maintained in STIHH conditions and treated with Que; fourth group (control group, LTNx + CMC) - rats maintained in $\mathrm{Nx}$ conditions for long-term and treated with CMC; fifth group (LTIHH + CMC) - rats maintained in (nu e baie calda) LTIHH conditions and treated with CMC; sixth group (LTIHH + Que) - rats maintained in LTIHH conditions and treated with Que. The Que was suspended in CMC $(0.01 \mathrm{~g} / \mathrm{ml})$ and orally administered through an intragastric tube, at a dose of $20 \mathrm{mg} / \mathrm{kg}$ once a day for 2 days and for 4 consecutive weeks, respectively, $30 \mathrm{~min}$ before each IHH exposure. The control groups were treated with vehicle $(0.6 \mathrm{ml}$ of CMC) through an intragastric tube. The body weight $(\mathrm{BW})$ of the rats was measured at the beginning and at the end of the experiment.

Twenty-four hours after the last exposure to normoxia or hypoxia, all rats were anesthetized by intraperitoneal administration of sodium pentobarbital $(60 \mathrm{mg} / \mathrm{rat})$ and sacrificed by cervical dislocation and the heart of each animal was quickly dissected. Next, the heart was removed immediately, washed with physiological serum (PS) to remove residual tissues and blood, weighed, placed into tubes, frozen, and stored at $-80{ }^{\circ} \mathrm{C}$. 
Preparation of biological samples

The frozen heart tissue samples were placed into plastic tubes, homogenized at a concentration of $100 \mathrm{mg}$ tissue per $\mathrm{ml}$ of $25 \mathrm{mM}$ buffer ( $\mathrm{pH}$ 7.4) composed of (in mmol/L): 10 Tris$\mathrm{HCl}, 137 \mathrm{NaCl}, 1 \mathrm{Na}_{2}$ EDTA, 0.5 dithiothreitol, and 250 sucrose on ice using a Polytron homogenizer (Brinkman Kinematica, Switzerland). The homogenate was centrifuged for $10 \mathrm{~min}$ at $9,000 \times g$, and the cellular debris was discarded. The supernatant was allocated into separate tubes, stored at $-80^{\circ} \mathrm{C}$, and used for biochemical assays.

\section{Measurement of biochemical markers of oxidative/nitrosative stress}

Because free radicals are highly reactive and have a very short lifetime, we indirectly measured their levels in the heart tissue homogenates by determining some products of lipid peroxidation and protein oxidation (protein carbonylation).

Estimation of lipid peroxidation. The lipids are one of the primary targets of reactive oxygen species (ROS). The peroxidation of lipids produces highly reactive aldehydes, including malondialdehyde (MDA). The MDA is a primary biomarker of free radical-mediated lipid damage and oxidative stress. The MDA levels were measured from the heart tissue homogenate using the fluorimetric method with 2-thiobarbituric acid (TBA) described by Conti's method (8). This method was used to obtain a spectrofluorimetric measurement of the color produced during the reaction of TBA with MDA at $535 \mathrm{~nm}$. MDA levels are expressed as nanomole per milligram protein (nmol MDA/mg protein).

Estimation of protein carbonylation. The protein carbonylation under ROS action was estimated from the heart tissue homogenate by measuring protein carbonyl (PC) group levels. The PC derivates that are produced through the protein oxidative damage were determined using the fluorimetric method with 2,4-dinitrophenyl-hydrazine (29). The readings were performed using a spectrophotometer at 355-390 nm and to calculate the remaining carbonyl fragments, the molar extinction coefficient with a value of $22,000 / \mathrm{M} / \mathrm{cm}$ was used. The levels of the carbonyl-derivative groups were expressed as nanomole per milligram of protein (nmol/mg protein).

The activities of some antioxidant enzymes, such as superoxide dismutase (SOD) and catalase (CAT), in the heart tissue homogenate were also assayed.

Estimation of SOD activity. The SOD activity was assayed using the nitroblue tetrazolium (NBT) method proposed by Flohe et al. (17). NBT was reduced to blue formazen by superoxide, which has a strong absorbance at $560 \mathrm{~nm}$. One unit (U) of SOD is defined as the amount of protein that inhibits the rate of NBT reduction by $50 \%$ in 1 min under the assay conditions. The SOD activity was expressed as units per milligram of protein (U/mg protein). Estimation of CAT activity. The CAT activity was assayed using the method proposed by Pippenger et al. (28). The method consists of following the change in absorbance of a solution of $\mathrm{H}_{2} \mathrm{O}_{2}(10 \mathrm{mM})$ in potassium phosphate buffer $(0.05 \mathrm{M}, \mathrm{pH}=7.4)$ at $240 \mathrm{~nm}$. One unit of CAT is defined as the amount of enzyme, which induces 0.43 reduction in absorbance at $25{ }^{\circ} \mathrm{C}$ for $3 \mathrm{~min}$. The CAT activity was also expressed as units per milligram of protein (U/mg protein).

By measuring the free radical production, the levels of nitrosative stress in the heart tissue homogenate were determined by measuring the NO and iNOS levels.

Estimation of nitrite plus nitrate (NOx) production. The NO production was indirectly detected by measuring the degradation products of $\mathrm{NO}$, the total nitrite level, after a prior conversion of nitrates into nitrites under the action of nitrate reductase. The NOx production was determined by measuring the nitrite, a stable end-product of NO metabolism using the 
Griess reaction (35). The supernatant of heart tissue homogenate was mixed with an equal volume of Griess reagent followed by spectrophotometric measurement at $543 \mathrm{~nm}$. Nitrite concentrations in the heart tissue homogenate were determined by comparison to a sodium nitrite standard curve. The NOx levels are presented as nanomole per milligram of protein (nmol/mg protein).

Estimation of $i N O S$. The level of iNOS in the heart tissue homogenate was measured using the commercially available enzyme-linked immunosorbent assay kits (R\&D Systems, Minneapolis, MN). The iNOS levels are expressed in nanogram per milligram of protein (ng/mg protein).

\section{Statistical analysis}

The statistical analysis was performed using the SPSS software package (version 17.0, SPSS Inc., Chicago, IL, USA). The data were reported as mean \pm standard deviation. One-way analysis of variance (ANOVA) was used to compare differences between groups, and two-way ANOVA for repeated measurements, followed by Tukey's multiple post-test comparisons, to compare the responses of Que and HH. Differences were considered significant, if $p<0.05$.

\section{Results}

Animal body weights and whole heart weights in the experimental groups after exposure to STIHH or LTIHH hypobaric hypoxia

The rats exposed to STIHH (STIHH + CMC group) showed insignificant changes in BW when compared to the rats maintained in Nx conditions for short-term (STNx + CMC group) (Table I). LTIHH exposure significantly increased final BW $(p<0.05)(\mathrm{LTIHH}+\mathrm{CMC}$ group). The treatment with Que of the rats exposed to LTIHH (LTIHH + Que) did not

Table I. Effects of short-term (2 days) and long-term (4 weeks) intermittent hypobaric hypoxia and quercetin administration on body weight (BW) and whole heart weight in control and experimental groups

\begin{tabular}{|l|c|c|c|c|c|c|}
\hline & $\begin{array}{c}\text { STNx } \\
\text { CMC }\end{array}$ & $\begin{array}{c}\text { STIHH + } \\
\text { CMC }\end{array}$ & $\begin{array}{c}\text { STIHH + } \\
\text { Que }\end{array}$ & $\begin{array}{c}\text { LTNx } \\
\text { CMC }\end{array}$ & $\begin{array}{c}\text { LTIHH + } \\
\text { CMC }\end{array}$ & $\begin{array}{c}\text { LTIHH + } \\
\text { Que }\end{array}$ \\
\hline Initial BW (g) & $278.8 \pm 11.40$ & $285 \pm 21.41$ & $286.5 \pm 20.24$ & $293 \pm 21.7$ & $275.7 \pm 18.44$ & $293 \pm 28.3$ \\
\hline Final BW (g) & $278.7 \pm 11.84$ & $287 \pm 21.15$ & $287.6 \pm 21.55$ & $291.7 \pm 22.72$ & $290.3 \pm 19.07$ & $295 \pm 29$ \\
\hline $\begin{array}{l}\text { Final whole heart } \\
\text { weight (g) }\end{array}$ & $0.79 \pm 0.15$ & $0.84 \pm 0.15$ & $0.86 \pm 0.11$ & $0.99 \pm 0.20$ & $1.16 \pm 0.16^{\mathrm{a}}$ & $0.9 \pm 0.09^{\mathrm{b}}$ \\
\hline $\begin{array}{l}\text { Final whole heart } \\
\text { weight/final BW } \\
\left(\times 10^{3}\right)\end{array}$ & $2.83 \pm 0.12$ & $2.92 \pm 0.07$ & $2.99 \pm 0.51$ & $3.39 \pm 0.88$ & $3.05 \pm 0.31^{\mathrm{a}}$ & $3.05 \pm 0.31^{\mathrm{a}}$ \\
\hline
\end{tabular}

STNx + CMC: normobaric normoxia conditions for short-term + CMC; STIHH + CMC: short-term intermittent hypobaric hypoxia + CMC; STIHH + Que: short-term intermittent hypobaric hypoxia + Que; LTNx + CMC: normobaric normoxia conditions for long-term + CMC; LTIHH + CMC: long-term intermittent hypobaric hypoxia + CMC; LTIHH + Que: long-term intermittent hypobaric hypoxia + Que. Results are mean \pm SD of 10 rats per each group. Statistically significant differences are indicated by the symbols: ${ }^{a} p<0.05$ vs. LTNx + CMC group; ${ }^{b} p<0.05$ vs. $\mathrm{LTIHH}+\mathrm{CMC}$ group 
significantly increase final $\mathrm{BW}(p>0.05)$ as compared to the control rats $(\mathrm{LTNx}+\mathrm{CMC}$ group) (Table I).

The final whole heart weight did not increase significantly following 2 days of exposure to IHH (STIHH + CMC group; Table I). After 4 weeks of exposure to IHH (LTIHH + CMC group), significant cardiac hypertrophy was observed due to increased final whole heart weight and final whole heart weight-to-final BW $(p<0.05$; Table I). Rats treated with Que and exposed for 4 weeks to IHH (LTIHH + Que group) showed a significant decrease in final whole heart weight and final whole heart weight-to-final BW $(p<0.05)$ as compared to the control rats (LTNx + CMC and LTIHH + CMC groups) (Table I).

Effects of Que on lipid peroxidation and proteins carbonylation in the heart tissue homogenate of rats exposed to STIHH or LTIHH

Level of lipid peroxidation. The damage of lipid molecules is indicated by the generation of MDA. The MDA level in the heart was significantly higher $(p<0.0001)$ after exposure of animals for either 2 days (STIHH + CMC group) or 4 weeks (LTIHH + CMC group) to IHH as compared to the control rats (STNx + CMC and LTNx + CMC groups, respectively). After 4 weeks of exposure to IHH (LTIHH + CMC group), the MDA level was higher compared to the 2 days of exposure to IHH (STIHH + CMC group), the difference was statistically significant $(p<0.0001)$ (Fig. 1a). Rats treated with Que and exposed for either 2 days (STIHH + Que group) or 4 weeks (LTIHH + Que group) to IHH showed significantly reduced MDA levels $(p<0.05)$ in both treated groups as compared to the control rats (STIHH + CMC and LTIHH + CMC groups, respectively) (Fig. 1a).

Level of protein oxidation. Reactive PC derivates have been measured as an index of tissue damage by oxidative stress. The effect of hypoxia on protein oxidation was measured by determining PC content in heart tissue homogenate. PC-derivative formation (PC groups) in the heart was also increased $(p<0.0001)$ in STIHH and LTIHH-exposed animals (STIHH + $\mathrm{CMC}$ and LTIHH + CMC groups) as compared to the control animals (STNx + CMC and LTNx + CMC groups, respectively) (Fig. 1b). The STIHH and LTIHH-exposed rats treated with Que (STIHH + Que and LTIHH + Que groups) showed significantly decreased PC levels $(p<0.0001)$ in the heart tissue as compared to the control STIHH and LTIHH-exposed animals (STIHH + CMC and LTIHH + CMC groups, respectively) (Fig. 1b). Que protected the heart tissue from protein oxidation induced by IHH exposure (STIHH + Que and LTIHH + Que groups); protection was statistically the most significant $(p<0.0001)$ in rats exposed to LTIHH (Fig. 1b).

Effects of Que on the activities of antioxidant enzymes in the heart tissue homogenate of rats exposed to STIHH or LTIHH

As the biomarkers of the antioxidant defenses, the activities of SOD and CAT in the heart were measured (Fig. 2).

The SOD activity was very significantly decreased $(p<0.0001)$ in the heart tissue homogenate after exposure of animals for either 2 days (STIHH + CMC group) or 4 weeks (LTIHH + CMC group) to IHH as compared to the control animals (STNx + CMC and LTNx + CMC groups, respectively), a decrease being statistically significant after exposure to LTIHH (LTIHH + CMC group) (Fig. 2a). Rats treated with Que and exposed to STIHH (STIHH + Que group) or LITHH (LTIHH + Que group) significantly augmented $(p<0.0001)$ SOD activity in the heart as compared to the control animals (STIHH + CMC and LTIHH + CMC groups, respectively) (Fig. 2a). 


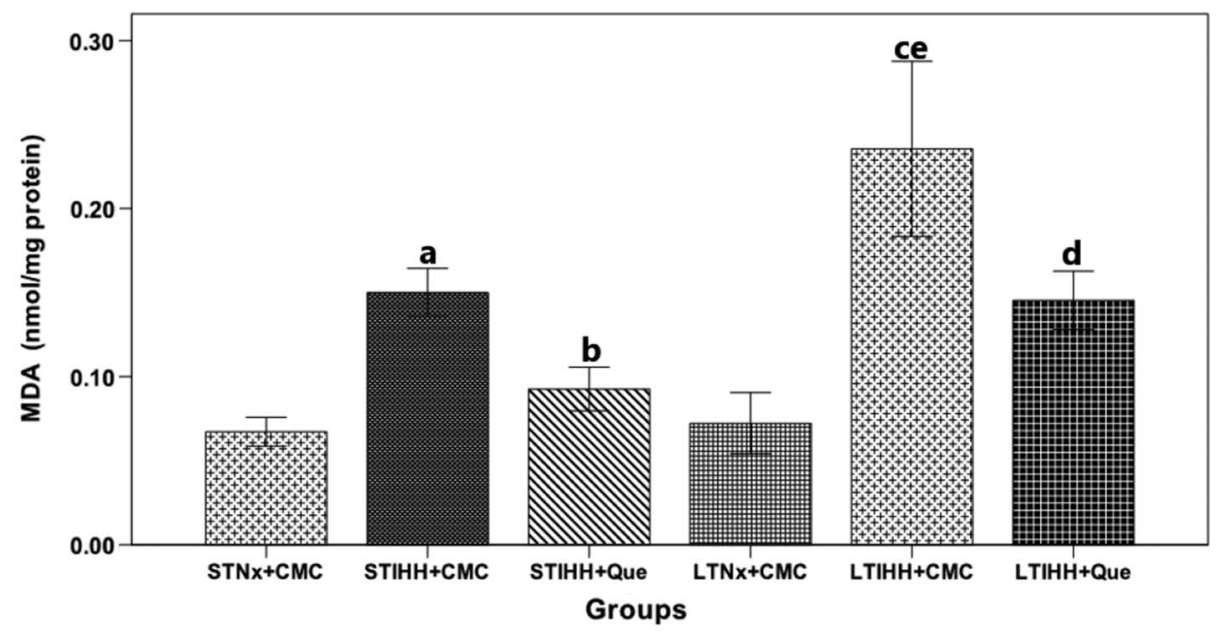

(a)

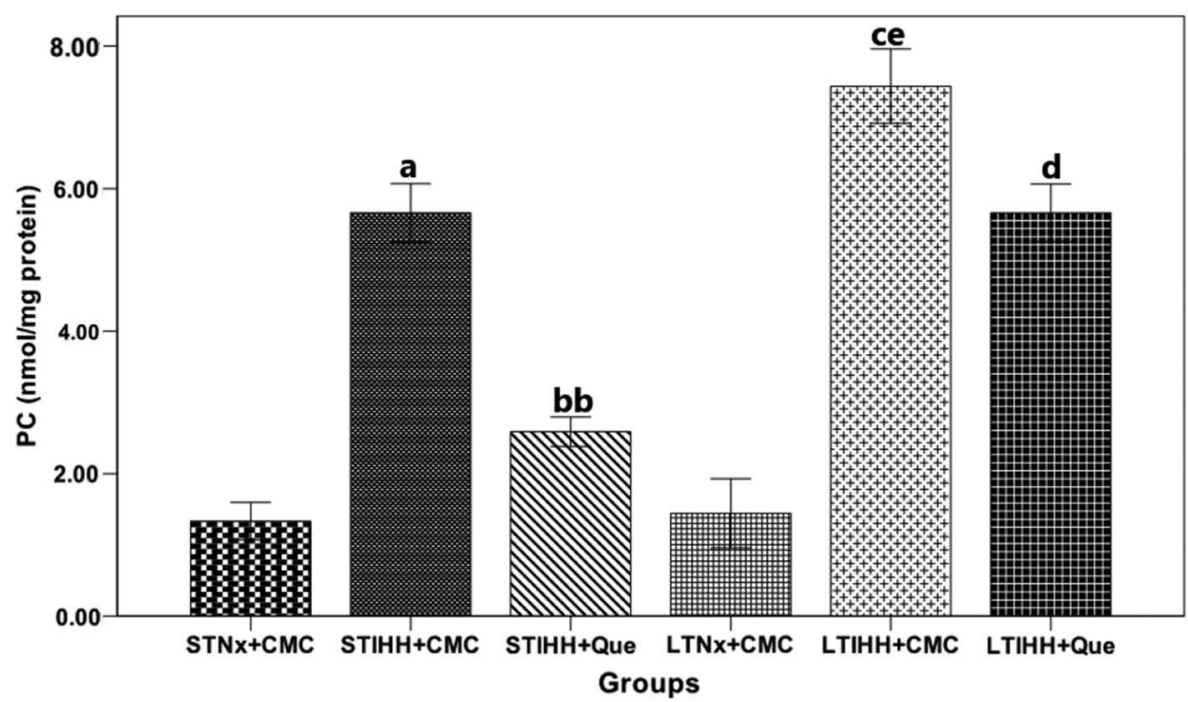

(b)

Fig. 1. The effects of quercetin on (a) lipid peroxidation (MDA) (nmol/mg protein) and (b) protein oxidation (protein carbonyl groups) (nmol/mg protein) levels in the heart tissue homogenates of control and experimental rats exposed to short-term or long-term intermittent hypobaric hypoxia. Results are the mean $\pm \mathrm{SD}$ for 10 animals in each group in the STNx + CMC, STIHH + CMC, STIHH + Que, LTNx+CMC, LTIHH + CMC, LTIHH + Que groups.

Statistically significant differences are indicated by the symbols: ${ }^{\mathrm{a}} p<0.0001$ vs. STNx $+\mathrm{CMC}$ group; ${ }^{\mathrm{b}} p<0.05$, ${ }^{\mathrm{bb}} p<0.0001$ vs. STIHH + CMC group; ${ }^{c} p<0.0001$ vs. LTNx + CMC group; ${ }^{\mathrm{d}} p<0.0001$ vs. LTIHH + CMC group; ${ }^{\mathrm{e}} p<0.0001 \mathrm{LTIHH}+\mathrm{CMC}$ group vs. STIHH $+\mathrm{CMC}$ group

The CAT activity was significantly reduced in the heart after exposure of rats for 2 days $(\mathrm{STIHH}+\mathrm{CMC}$ group; $p<0.05)$ or 4 weeks $(\mathrm{LTIHH}+\mathrm{CMC}$ group; $p<0.0001)$ to IHH as compared to the control groups (STNx + CMC and LTNx + CMC groups, respectively), a decrease being statistically significant after exposure to LTIHH (LTIHH + CMC group) (Fig. 2b). Que administration has significant effects on the increase of CAT activity in the 


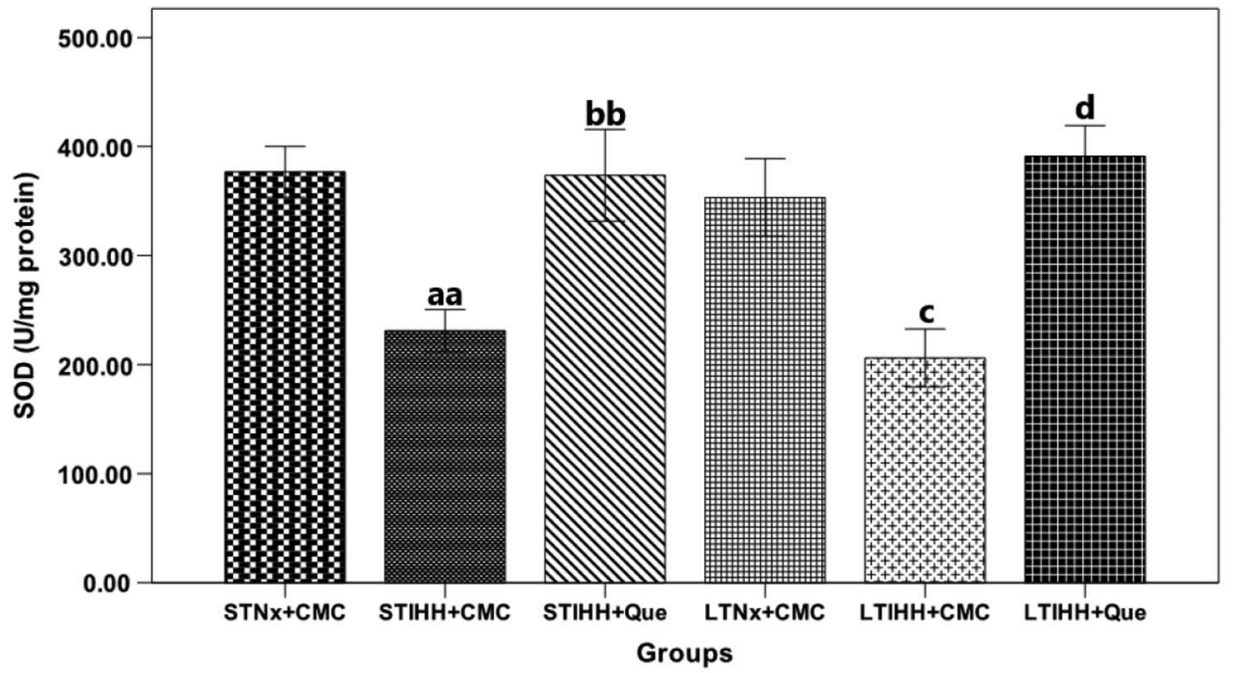

(a)

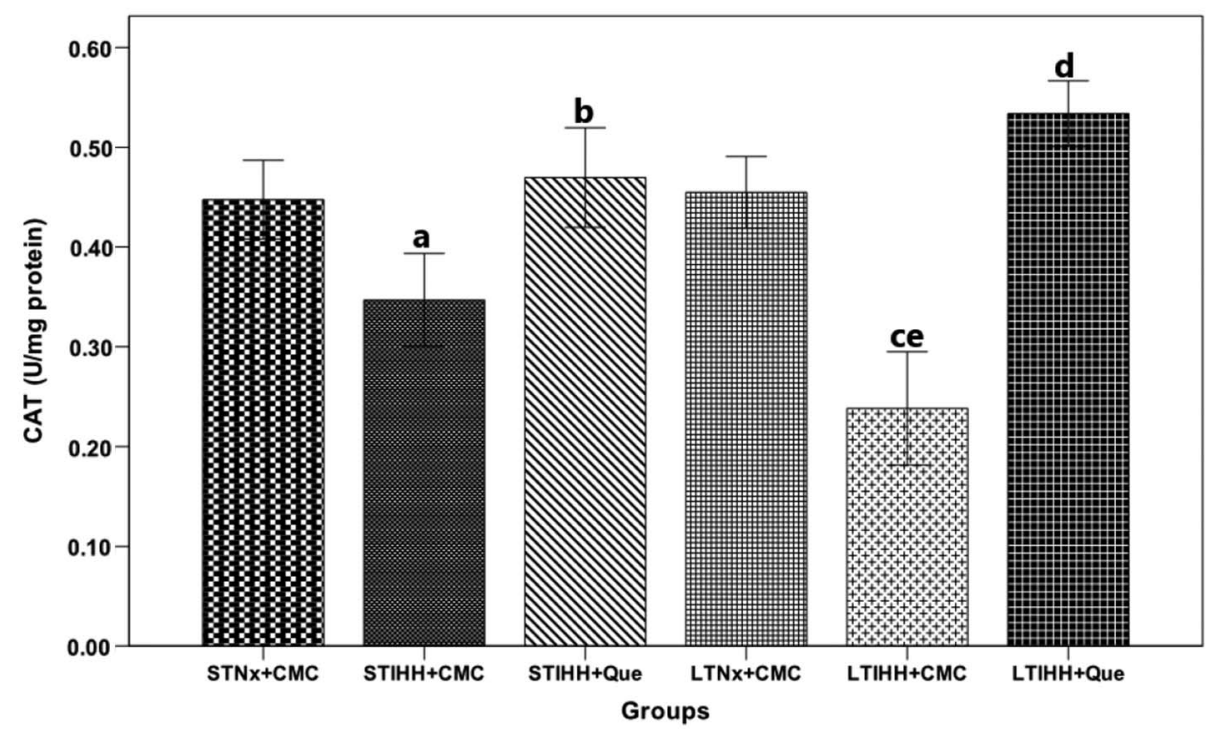

(b)

Fig. 2. The effects of quercetin on the level of (a) superoxide dismutase (SOD) (U/mg protein) and (b) catalase (CAT) (U/mg protein) activities in the heart tissue homogenates of control and experimental rats exposed to short-term or long-term intermittent hypobaric hypoxia. Results are the mean \pm SD for 10 animals in each group in the STNx +

$\mathrm{CMC}, \mathrm{STIHH}+\mathrm{CMC}, \mathrm{STIHH}+$ Que, LTNx + CMC, LTIHH + CMC, LTIHH + Que groups. Statistically significant differences are indicated by the symbols: ${ }^{\mathrm{a}} p<0.005,{ }^{\mathrm{aa}} p<0.0001 \mathrm{vs}$. STNx $+\mathrm{CMC}$ group; ${ }^{\mathrm{b}} p<0.005$, ${ }^{\mathrm{bb}} p<0.0001$ vs. STIHH + CMC group; ${ }^{\mathrm{c}} p<0.0001$ vs. LTNx + CMC group; ${ }^{\mathrm{d}} p<0.0001$ vs. LTIHH + CMC group; $\mathrm{e}_{p}<0.005 \mathrm{LTIHH}+\mathrm{CMC}$ group vs. STIHH + CMC group

heart of rats exposed for 2 days (STIHH + Que group; $p<0.005)$ or 4 weeks (LTIHH + Que group; $p<0.0001)$ as compared to the control animals (STIHH + CMC and LTIHH groups, respectively) (Fig. 2b). 


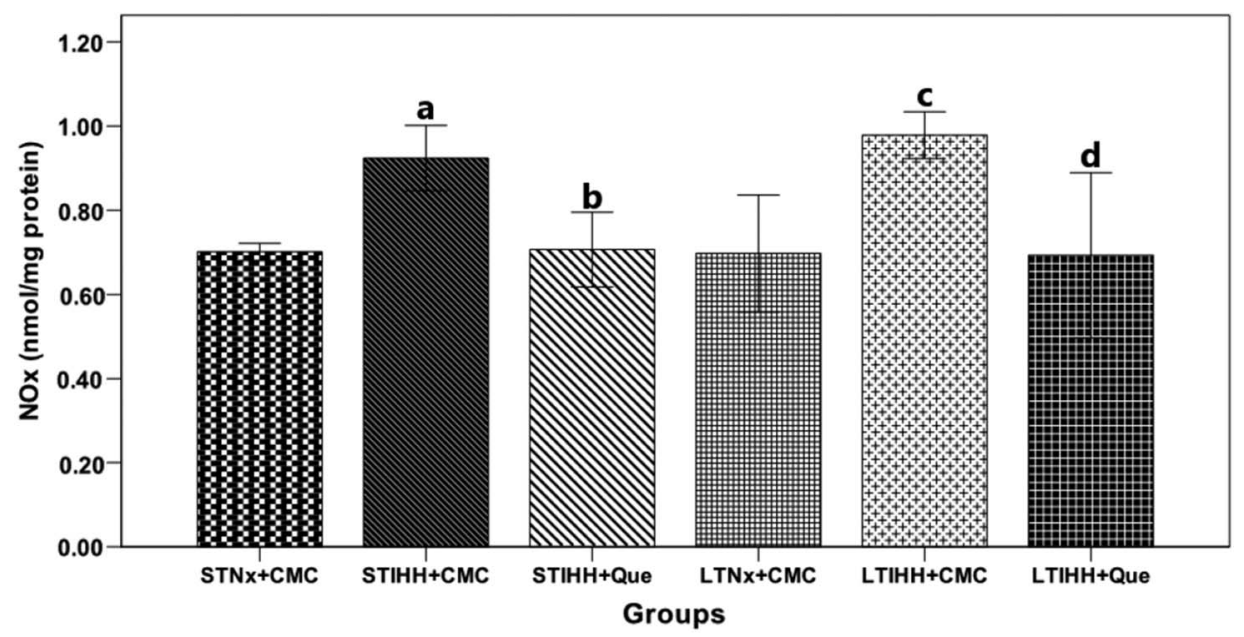

(a)

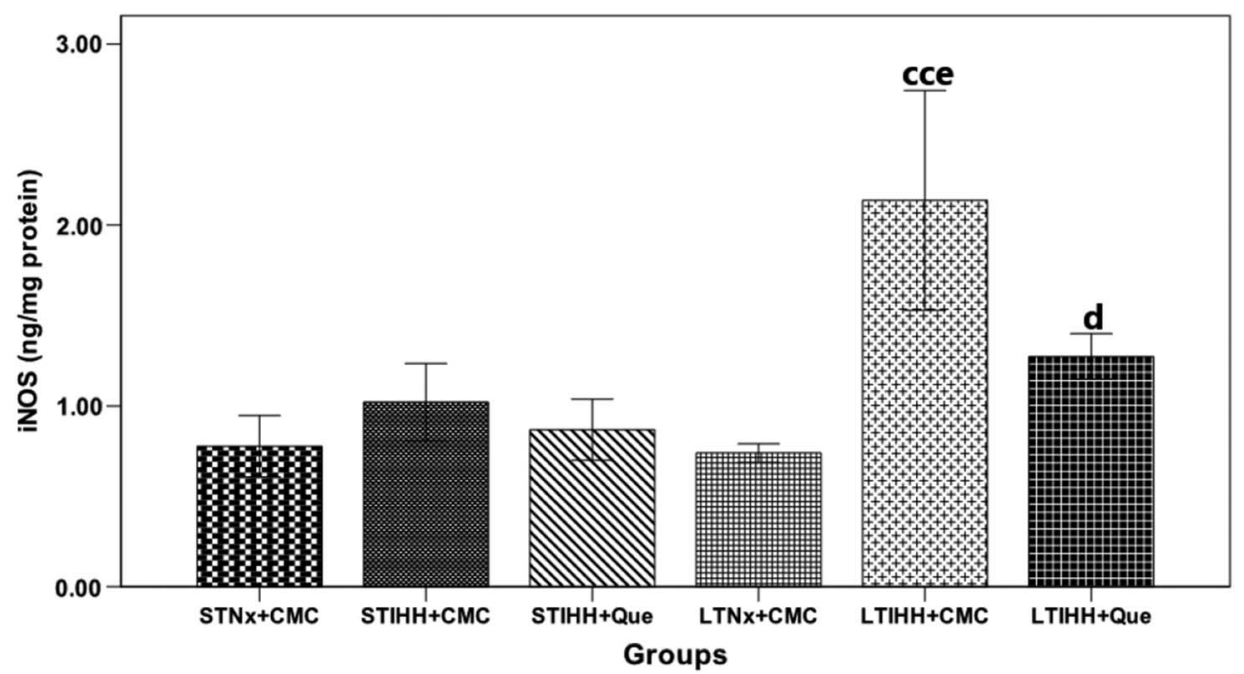

(b)

Fig. 3. The effects of quercetin on the level of (a) nitrite plus nitrate production (NOx) (nmol/mg protein) and

(b) inducible nitric oxide synthase (iNOS) (ng/mg protein) in the heart tissue homogenates of control and experimental rats exposed to short-term or long-term intermittent hypobaric hypoxia. Results are the mean $\pm \mathrm{SD}$ for 10 animals in each group in the STNx + CMC, STIHH + CMC, STIHH + Que, LTNx + CMC,

$\mathrm{LTIHH}+\mathrm{CMC}$, LTIHH + Que groups. Statistically significant differences are indicated by the symbols: ${ }^{\mathrm{a}} p<0.05$ vs. STNx $+\mathrm{CMC}$ group; ${ }^{\mathrm{b}} p<0.05$ vs. STIHH $+\mathrm{CMC}$ group; ${ }^{\mathrm{c}} p<0.005,{ }^{\mathrm{cc}} p<0.0001$ vs. LTNx + CMC group; ${ }^{\mathrm{d}} p<0.005$ vs. LTIHH + CMC group; ${ }^{\mathrm{e}} p<0.0001 \mathrm{LTIHH}+\mathrm{CMC}$ group vs. $\mathrm{STIHH}+\mathrm{CMC}$ group

Que protected the heart tissue from the oxidative stress induced by IHH (STIHH + Que and LTIHH + Que groups) exposure, protection evidenced by the increased activities of antioxidant enzymes (SOD and CAT; Fig. 2). 
Effects of Que on nitrosative stress in the heart tissue homogenate of rats exposed to STIHH or LTIHH

Nitrite production. The levels of nitrites (NOx) were measured as indicators of NO production, which is one of the most important regulatory factors in the cardiovascular system. After 2 days of exposure to IHH (STIHH + CMC group), the nitrite levels in the heart were higher $(p<0.05)$ as compared to the control rats (STNx + CMC group). Nitrites levels were increased appreciably $(p<0.005)$ after 4 weeks of exposure to IHH (LTIHH + CMC group) as compared to the control group (LTNx + CMC group) (Fig. 3a). Que administration that is determined significantly decreased $(p<0.05)$ the levels of nitrites in the heart of the rats exposed for 2 days to IHH (STIHH + Que group) as compared to the control group (STIHH + CMC group), the decrease being more significant $(p<0.005)$ in the rats exposed to LTIHH and treated with Que (LTIHH + Que group) as compared to the control group (LTIHH + Que group) (Fig. 3a).

iNOS protein expression. The iNOS level in the heart tissue homogenate of the rats exposed to IHH for 4 weeks (LTIHH + CMC group) was significantly higher $(p<0.0001)$ than that of the control rats (LTNx + CMC group). After 2 days of exposure to IHH, the iNOS level in the heart tissue homogenate was not significantly higher $(p>0.05)$ than that of the control rats (STNx + CMC group) (Fig. 3b). The rats exposed to IHH for 2 days and treated with Que (STIHH + Que group) showed non-significant decreases in iNOS levels $(p>0.05)$ in heart tissue as compared to the control rats (STIHH + CMC group). A significant decrease $(p<0.005)$ of the iNOS levels was observed in the rats exposed to IHH for 4 weeks and treated with Que (LTIHH + Que group) as compared to their control rats (LTIHH + CMC group) (Fig. 3b).

\section{Discussion}

This study represents the attempt to observe how the heart reacts to STIHH or LTIHH stress and the effects of supplementation with natural antioxidants (Que) using an in vivo model. The findings of this study were: (1) cardiac hypertrophy was found in rats after 4 weeks of IHH exposure. However, no significant changes of heart weight-to-body weight ratio were found in the rat's heart after 2 days of IHH exposure; (2) Que administration significantly decreased the heart weight-to-body weight ratio after a LTIHH exposure; (3) 4 weeks of exposure to IHH caused a marked increase in ROS generation, lipid peroxidation, and protein oxidation; (4) the NO production and iNOS level increased significantly after 4 weeks of IHH exposure; (5) enzymatic antioxidant levels decreased in heart tissue after IHH exposure; and (6) Que supplementation attenuates RONS generation after IHH exposure. Our major findings imply that changes of cardiac hypertrophy under IHH exposure are tightly time course-dependent, and STIHH exposure and Que administration can provide protection from oxidative/nitrosative stress in hypoxic heart, thus representing a therapeutic strategy in heart protection in ischemia/reperfusion injuries.

$\mathrm{HA}$ is characterized by $\mathrm{HH}$, which is considered a physiological stress characterized by increasing oxidative/nitrosative stress, consequently causing potential damage to lipids, proteins, and DNA (33). Oxidative/nitrosative stress increased production of RONS and decreased intracellular antioxidant defense, thus causing an imbalance in the redox status of the cell $(12,13)$. Recent in vitro and animal research have demonstrated the involvement of RONS in impairment of the cardiovascular system in response to various heart stressors $(2,6)$. Exposure to IHH increases RONS generation in the myocardium, which appears to be 
the main cause of myocardial damage. RONS produced in excess in the myocardium cause cardiomyocyte injury, apoptosis, and cell necrosis (42). In this study, cardiac hypertrophy was observed after long-term exposure to $\mathrm{IHH}$, as evidenced by the significant increase in the heart weight-to-body weight ratio. After 2 days of IHH exposure, the rats showed a less significant cardiac hypertrophy. Our results are in accordance with recent data; the main consequences of adaptation to $\mathrm{HH}$ are an increased cardiac resistance to acute ischemic injury and myocardial hypertrophy (33). In the hypoxic heart, cardiomyocyte apoptosis occurs with the distortion of cardiac architecture. Therefore, after long-term exposure to IHH, myocardial fibrosis and ventricular hypertrophy with myocardial dysfunction and heart failure occur. On the contrary, short-term exposure to IHH provides myocardial protection against myocardial injury. The protective effects would promote myocardial contractile recovery of cardiac function in ischemia/reperfusion injury, limiting myocardial infarction, and cardiac arrhythmias (36-39). The rats treated with Que and exposed to LTIHH showed a significant decrease in the heart weight-to-body weight ratio, demonstrating cardioprotective effects. These data complete our previous studies and results of other researchers that showed the cardioprotective effects of Que in ischemia/reperfusion injury $(6,14,15)$.

In this study, we investigated the oxidative/nitrosative stress induced in the rat's heart by IHH exposure and the possible cardioprotective effects of Que supplementation. Our data clearly suggest that IHH exposure determines a significant RONS generation in heart tissue. RONS generation after IHH exposure caused further damage to cardiomyocyte membrane composition.

This study demonstrates that STIHH or LTIHH exposure induces increase in oxidative stress in the heart, which is proven by the growth of lipid peroxidation and protein oxidation in the heart tissue homogenate, as previously reported (16).

Lipids undergo peroxidation in the presence of RONS and oxygen. MDA, a major oxidant product of membrane-peroxidized polyunsaturated fatty acids, is an indicator for oxidative stress. MDA level indirectly reflects the severity of peroxidation damage in body cells attacked by free radicals. It is one of the indicators to evaluate organism ischemic injury. MDA level in STIHH group had changed significantly, which indicated that lipid peroxidation occurred in early HH stage. Protein modifications caused by RONS include formation of carbonyls, dityrosine, and nitrated and chlorinated tyrosines (13). Our results are in accordance with the results obtained by other researchers $(16,42)$. After exposure to LTIHH, the products of the lipid and protein oxidative damage in the heart tissue homogenate increased more significantly ( $p<0.0001)$, as opposed to exposure to STIHH. These studies demonstrated that Que plays a protective role in the heart of rats exposed to IHH as a result of products of lipid peroxidation, demonstrated by lower MDA content, and protein oxidation, demonstrated by decreasing PC content in the heart tissue homogenate. The effect of Que was corroborated by changes in heart weight. This view of protective role of Que in heart tissue is supported by our previous studies in which we observed the restoration of cardiac architecture in rats exposed to LTIHH after treatment with Que (15).

To correlate the results obtained from the investigation of oxidative stress after STIHH and LTIHH exposure, we monitored some antioxidant enzymes' activities such as SOD and CAT in the heart tissue, enzymes responsible for scavenging RONS. SOD is one very important enzymatic scavenger as it plays a critical role in protecting mitochondria from superoxide anions generated during respiration. It accelerates the dismutation of superoxide anions into hydrogen peroxide, which can be regarded as a primary defense in preventing the generation of free radicals (10). CAT is localized intracellularly in the mitochondria and 
peroxisomes and is responsible for scavenging the hydrogen peroxide resulting from superoxide anions after SOD action $(13,19)$. In this study, we have shown that hypoxia is associated with decreased SOD and CAT concentrations in the heart of rats exposed to IHH for either 2 days or 4 weeks. Que supplementation in rats exposed to IHH significantly increased the activity of antioxidant enzymes (SOD and CAT) in the heart tissue, which then may help to protect the heart from oxidative damage. The accumulation of damaged products is probably partially prevented by the elevated activities of antioxidant enzymes (SOD and CAT) in the heart. These results are in concordance with other reports that demonstrated antioxidative actions of Que in different tissues $(2-4,6,14,15,24,32)$.

To fully elucidate the effects of IHH exposure in the animal, we studied NO, one of the main candidates to accord the cardioprotection effect at HA $(33,39,42)$. This study demonstrated that 2 days of IHH exposure increased NO production. Excessive NO production after STIHH exposure had the role of protecting the hypoxic heart. In the heart, after exposure to $\mathrm{HH}, \mathrm{NF}-\kappa \mathrm{B}$ is activated, increasing the expression of HIF-1 $\alpha$, which in turn expands the generation of NO $(30,41)$. Excessive NO production in the hypoxic heart is responsible for increasing erythropoiesis, vascularization, and vasodilatation in the heart and therefore cardioprotection. After exposure to LTIHH, the NO production and iNOS level in the heart tissue homogenate increased more significantly. NO in high concentration exerts pathological effects by rapidly interacting with superoxide anion; even low concentrations can form peroxynitrite (9). The peroxynitrite radical causes lipid peroxidation, apoptosis, DNA alterations, nitration, and oxidation of proteins, causing destruction determined in the cardiomyocytes (31).

This study also reports that the source of increased NO production after exposure to LTIHH may be iNOS, showing an increase in nitrosative stress in the heart responsible for cardiac damage. In our research, after 2 days of IHH exposure, there was a non-significant increase in the iNOS production in the heart, but after 4 weeks of IHH exposure, the concentrations of iNOS increased very significantly in the heart tissue. These results confirm that exposure of the heart to STIHH has cardioprotective effects, whereas long exposure to IHH causes cardiac damage.

In this study, the rats treated with Que and exposed to LTIHH showed a significant decrease in the NO produced and in the iNOS level of the heart. The results from this study also suggest that Que modulates its cardioprotective effects by the enhancement of NO bioavailability through the metabolism of superoxide anions and/or its reactive metabolites and by directly scavenging ROS and free radicals and activating antioxidant enzymes $(4,15)$, data which were in agreement with other findings recently reported by other authors $(4,11$, 20,21). Recent research shows that Que inhibits the NADPH oxidase complex that mediates the synthesis of superoxide anion and inhibits iNOS expression and thus decreases the concentration of NO, an effect mediated by inhibition of nuclear translocation of NF- $\mathrm{KB}(21)$.

The present results showed that STIHH exposure induces cardioprotection in hypoxic heart, and LTIHH exposure induces the growth of oxidative/nitrosative stress in the heart, which is ultimately responsible for cardiac damage. Que supplementation exerted a protective effect against the markers of cardiac cell stress.

\section{Conclusions}

In conclusion, our data demonstrate that Que exerts cardioprotective effects in the heart of rats exposed to IHH. These beneficial actions of Que were mediated by antioxidant protection of oxidative stress coupled with neutralizing reactive metabolites of NO. Furthermore, these 
results suggest that Que administration before exposure to hypoxia may have beneficial effects against cardiac damage circumscribed by oxidative/nitrosative stress.

\section{Acknowledgements}

The authors gratefully acknowledge Remus Moldovan for animal handling. They would also like to thank Nicoleta Decea for her help regarding oxidative/nitrosative stress parameters' assessment.

\section{Conflict of interest}

The authors declare no conflict of interest.

\section{REFERENCES}

1. Aktan F: iNOS-mediated nitric oxide production and its regulation. Life Sci. 75, 639-653 (2004)

2. Annapurna A, Reddy CS, Akondi RB, Rao SR: Cardioprotective actions of two bioflavonoids, quercetin and rutin, in experimental myocardial infarction in both normal and streptozotocin-induced type I diabetic rats. J. Pharm. Pharmacol. 61, 1365-1374 (2009)

3. Bakhshaeshi M, Khaki A, Fathiazad F, Khaki AA, Ghadamkheir E: Anti-oxidative role of quercetin derived from Allium cepa on aldehyde oxidase (OX-LDL) and hepatocytes apoptosis in streptozotocin-induced diabetic rat. Asian Pac. J. Trop. Biomed. 2, 528-531 (2012)

4. Boots AW, Haenen GR, Bast A: Health effects of quercetin: from antioxidant to nutraceutical. Eur. J. Pharmacol. 585, 325-337 (2008)

5. Chandel NS, Maltepe E, Goldwasser E, Mathieu CE, Simon MC, Schumacker PT: Mitchondrial reactive species trigger hypoxia induced transcription. Proc. Natl. Acad. Sci. U. S. A. 95, 11715-11720 (1998)

6. Chen YW, Chou HC, Lin ST, Chen YH, Chang YJ, Chen L, Chan HL: Cardioprotective effects of quercetin in cardiomyocyte under ischemia/reperfusion injury. Evid. Based Complement. Alternat. Med. 2013, 364519 (2013)

7. Chis IC, Baltaru D, Marton A, Maier M, Muresan A, Clichici S: Effects of quercetin and chronic (training) exercise on oxidative stress status in animals with streptozotocin-induced diabetes. Bull. USAMV-CN Vet. Med. 70, 31-39 (2013)

8. Conti M, Morand PC, Levillain P: Improved fluorimetric determination of malonaldehyde. Clin. Chem. 37, 1273-1275 (1991)

9. Daff S: NO synthase: structure and mechanisms. Nitric Oxide 23, 1-11 (2010)

10. Di Naso FC, Simoes Dias A, Porawski M, Marroni NA: Exogenous superoxide dismutase: action on liver oxidative stress in animals with streptozotocin-induced diabetes. Exp. Diab. Res. 2011, 754132 (2011)

11. Dias AS, Porawski M, Alonso M, Marroni N, Collado PS, González-Gallego J: Quercetin decreases oxidative stress, NF-kappaB activation, and iNOS over expression in liver of streptozotocin-induced diabetic rats. J. Nutr. 135, 2299-2304 (2005)

12. Dosek A, Ohmo H, Acs Z, Taylor AW, Radak Z: High altitude and oxidative stress. Respir. Physiol. Neurobiol. 158, 128-131 (2007)

13. Droge W: Free radicals in the physiological control and cell function. Physiol. Rev. 82, 47-95 (2002)

14. Dumitrovici A, Bolfa PF, Mureşan A, Maier M, Chiş IC: Short-term versus long-term intermittent hypobaric hypoxia on cardiac fibrosis and cardioprotective effects of natural antioxidants supplementation in rat hearts. Bull. USAMV-CN Vet. Med. 70, 56-65 (2013)

15. Dumitrovici A, Chiş IC, Mureşan A, Marton A, Moldovan R, Vlad D, Borza G, Bolfa P: Quercetin, Lycium barbarum and Chitosan reverse the effects of hypobaric hypoxia and exert cardioprotective effects in rats. Fiziologia (Physiology) 23, 18-22 (2013)

16. Farías JG, Zepeda AB, Calaf GM: Melatonin protects the heart, lungs and kidneys from oxidative stress under intermittent hypobaric. Biol. Res. 45, 81-85 (2012)

17. Flohe L, Becker R, Brigelius R, Lengfelder E, Ötting F (1990): Convenient assays for superoxide dismutase. In: CRC Handbook of Free Radicals and Antioxidants in Biomedicine, vol III, ed Miquel J, CRC Press, Boca Raton, pp. 287-293

18. Formagio ASN, Kassuya CAL, Neto FF, Volobuff CRF, Iriguchi EKK, Vieira M, Foglio MA: The flavonoid content and antiproliferative, hypoglycaemic, anti-inflammatory and free radical scavenging activities of Annona dioica St. Hill. BMC Complement. Altern. Med. 13, 14-22 (2013) 
19. Hanasaki Y, Ogawa S, Fukui S: The correlation between active oxygens scavenging and antioxidative effects of flavonoids. Free Radic. Biol. Med. 16, 845-850 (1994)

20. Jeong SM, Kang MJ, Choi HN, Kim JH, Kim JI: Quercetin ameliorates hyperglycemia and dyslipidemia and improves antioxidant status in type 2 diabetic db/db mice. Nutr. Res. Pract. 6, 201-207 (2012)

21. Jo HY, Kim Y, Nam SY, Lee BJ, Kim YB, Yun YW, Ahn B: The inhibitory effect of quercetin gallate on iNOS expression induced by lipopolysaccharide in Balb/c mice. J. Vet. Sci. 9, 267-272 (2008)

22. Jung F, Palmer LA, Zhou N, Johns RA: Hypoxic regulation of inducible nitric oxide synthase via hypoxia inducible factor-1 in cardiac myocytes. Circ. Res. 86, 319-325 (2000)

23. Kaluz S, Kaluzova M, Stanbridge EJ: Regulation of gene expression by hypoxia: integration of the HIFtransduced hypoxic signal at the hypoxia-responsive element. Clin. Chim. Acta 395, 6-13 (2008)

24. Kanter M, Aktas C, Erboga M: Protective effects of quercetin against apoptosis and oxidative stress in streptozotocin-induced diabetic rat testis. Food Chem. Toxicol. 50, 719-725 (2012)

25. Lee SD, Kuo WW, Wu CH, Lin YM, Lin JA, Lu MC, Yang AL, Liu JY, Wang SG, Liu CJ, Chen LM, Huang CY: Effects of short- and long-term hypobaric hypoxia on Bcl2 family in rat heart. Int. J. Cardiol. 108, 376-384 (2006)

26. Lin YM, Huang SK, Wang HF, Chen LM, Tsai FJ, Hsu HH, Kuo CH, Wang PS, Huang CY, Lee SD: Short-term versus long-term intermittent hypobaric hypoxia on cardiac fibrosis and Fas death receptor dependent apoptotic pathway in rat hearts. Chin. J. Physiol. 51, 308-316 (2008)

27. Martinez-Flores S, Gutierez MB, Sanchez-Campos S, Gonzales-Gallego J, Tunon MJ: Quercetin prevents nitric oxide production and nuclear factor kappa B activation in interleukin-1 $\beta$-activated rat hepatocytes. J. Nutr. 135, 1359-1365 (2005)

28. Pippenger CE, Browne RW, Armstrong D (1998): Regulatory antioxidant enzymes. In: Free radical and antioxidant protocols. Methods in molecular biology, vol 108, ed Armstrong D, Humana Press, Totowa, pp. 299-313

29. Reznick AZ, Packer L: Oxidative damage to proteins: spectrophotometric method for carbonyl assay. Methods Enzymol. 233, 347-357 (1994)

30. Romero RM, Canuelo A, Siles E, Oliver FJ, Lara ME: Nitric oxide modulates hypoxia-inducible factor-1 and poly (ADP-ribose) polymerase-1 cross talk in response to hypobaric hypoxia. J. Appl. Physiol. 112, 816-823 (2012)

31. Rus A, Peinado MA, Castro L, Del Moral MI: Lung eNOS and iNOS are reoxygenation time-dependent upregulated after acute hypoxia. Anat. Rec. (Hoboken) 293, 1089-1098 (2010)

32. Sarkar A, Angeline MS, Anand K, Ambasta RK, Kumar P: Naringenin and quercetin reverse the effect of hypobaric hypoxia and elicit neuroprotective response in the murine model. Brain Res. 24, 59-70 (2012)

33. Singh M, Thomas P, Shukla D, Tulsawani R, Saxena S, Bansal A: Effect of subchronic hypobaric hypoxia on oxidative stress in rat heart. Appl. Biochem. Biotechnol. 169, 2405-2019 (2013)

34. Tang D, Dong Y, Ren H, Li L, He C: A review of phytochemistry, metabolite changes, and medicinal uses of the common food mung bean and its sprouts (Vigna radiata). Chem. Cent. J. 8, 4 (2014)

35. Titherage MA (1998): The enzymatic measurement of nitrate and nitrite. In: Nitric oxide protocols. Methods in molecular biology, vol 100, ed Titherage MA, Humana Press, Totowa, pp. 83-91

36. Wang ZH, Cai XL, Wu L, Yu Z, Liu JL, Zhou ZN, Liu J, Yang HT: Mitochondrial energy metabolism plays a critical role in the cardioprotection afforded by intermittent hypobaric hypoxia. Exp. Physiol. 97, 1105-1118 (2012)

37. Wang ZH, Chen YX, Zhang CM, Wu L, Yu Z, Cai XL, Guan Y, Zhou ZN, Yang HT: Intermittent hypobaric hypoxia improves postischemic recovery of myocardial contractile function via redox signaling during early reperfusion. Am. J. Physiol. Heart. Circ. Physiol. 301, 1695-16705 (2011)

38. Zhang Y, Yang HT, Zhou ZN: Cardioprotection of intermittent hypoxia. Acta Physiol. Sin. 59, 601-613 (2007)

39. Zhang Y, Zhong N, Zhou ZN: Effects of chronic intermittent hypobaric hypoxia on the L-type calcium current in rat ventricular myocytes. High Alt. Med. Biol. 11, 61-67 (2011)

40. Zhong N, Zhang Y, Zhu HF, Wang JC, Fang QZ, Zhou ZN: Myocardial capillary angiogenesis and coronary flow in ischemia tolerance rat by adaptation to intermittent high altitude hypoxia. Acta Pharmacol. Sin. 23, 305-310 (2002)

41. Xie QW, Kashiwabara Y, Nathan C: Role of transcription factor NF-kB/Rel in induction of nitric oxide synthase. J. Biol. Chem. 269, 4705-4708 (1994)

42. Yin X, Zheng Y, Liu Q, Cai J, Cai L: Cardiac response to chronic intermittent hypoxia with a transition from adaptation to maladaptation: the role of hydrogen peroxide. Oxid. Med. Cell. Longev. 2012, 569520 (2012) 